\title{
STUDY OF CHILDREN WITH RECURRENT PNEUMONIA ADMITTED IN A TERTIARY HOSPITAL
}

\author{
Rijal P, Lama L, Shrestha S, Kakshapati P, Nayak R
}

Department of Pediatrics, Nepal Medical College Teaching Hospital, Attarkhel, Gokarneshwor-8, Kathmandu, Nepal.

\begin{abstract}
Recurrent pneumonia usually occurs due to an underlying disorder that negatively affects local or systemic defence mechanisms. The aim of this study was to find out the prevalence and risk factors associated with recurrent pneumonia in children and to determine possible strategic plan needed for better clinical outcome. Children between 2 months to 15 years old who had a history of 2 or more episodes of pneumonia per year, or 3 or more episodes in a life time were investigated prospectively at Nepal Medical College Teaching Hospital. Out of 653 children admitted for pneumonia, 74(11.3\%\%) met the criteria for recurrent pneumonia. Among 74 children with recurrent pneumonia, underlying risk factors was demonstrated in 65 patients (87.8\%). Most common underlying diseases were aspiration syndrome in $21.6 \%$ patients, congenital heart disease in $13.5 \%$ patients and bronchial asthma in $12.1 \%$ children. No predisposing illness could be demonstrated in $12.1 \%$ patients. Approximately 1 in 9 children with pneumonia in our hospital had recurrent pneumonia. Aspiration syndrome was the most common underlying illnesses for undiagnosed recurrent pneumonia in children.
\end{abstract}

\section{KEYWORDS}

Aspiration syndrome, bronchiectasis, immune deficiency disorders, recurrent pneumonia

\section{CORRESPONDING AUTHOR}

Dr. Prashant Rijal,

Assistant Professor, Department of Pediatrics, Nepal Medical College Teaching Hospital, Attarkhel, Gokarneshwor-8, Kathmandu, Nepal.

Email: prashant_rijal@hotmail.com 


\section{INTRODUCTION}

Pneumonia is an acute inflammation of the lung parenchyma that develops due to both infectious and non-infectious causes. Pneumonia is a major problem in children; the World Health Organization (WHO) estimated that pneumonia occurred in approximately 156 million children (151 million in developing countries and 5 million in developed countries). ${ }^{1}$ Recurrent pneumonia (RP) (at least 2 pneumonia episodes in 1-year period or more than 3 episodes at any time with radiographic clearing between episodes) was diagnosed in 6.4-9.0\% of children with pneumonia. ${ }^{2-4}$ Recurrent pneumonia usually result from deficiencies in the local pulmonary or systemic host defences or from underlying disorders that modify the lung defence. Therefore, paediatricians should investigate this possible underlying causes. ${ }^{5}$ There are limited data on the underlying diseases predisposing to recurrent pneumonia in children. In addition, few studies have addressed this problem in the developing world. ${ }^{6-8}$ The potential burden of $\mathrm{RP}$ on the health care system, in terms of frequent hospitalizations, is significant. Early recognition and management of the underlying cause is expected to reduce the number of pneumonia related hospital admissions, morbidity and mortality. Especially in the developing countries, increasing studies of epidemiology to recurrent pneumonia will ease preventative and therapeutic approach. The purpose of this study is to determine the prevalence and risk factors associated with recurrent pneumonia in Nepalese children in a tertiary care centre. Hence, we can make strategic plan to reduce the morbidity and mortality due to recurrent pneumonia.

\section{MATERIALS AND METHODS}

It is an observational cross-sectional study conducted at Nepal Medical College Teaching Hospital, Attarkhel, Kathmandu, Nepal. The hospital is situated at Gokarneshwor-8, Nepal about $11 \mathrm{~km}$. northeast of Kathmandu city. All patients with diagnosis of recurrent pneumonia admitted in pediatric ward of NMCTH during July 2013 to June 2018 were included in the study.

Patients who met the diagnostic criteria for RP were included in the study. Pneumonia was diagnosed based on the presence of tachypnea (with a respiratory rate of $\geq 60 / \mathrm{min}$ for infants aged $<2$ months, $\geq 50 /$ min for infants aged 2-11 months, $\geq 40 / \mathrm{min}$ for infants aged $12-60$ months) and $\geq 30$ in children more than 5 years of age, laboured breathing, bronchial breath sounds or crackles on chest auscultation and/ or radiological findings (pulmonary consolidation or infiltrate). ${ }^{9}$ Children with persistent pneumonia and outpatient department (OPD) patients were excluded from the study. Patient demographics (gender, age at diagnosis of RP, age at first episode, number of episodes per year, and number of episodes prior to diagnosis of RP) and details pertaining to the aetiology of and risk factors for RP (presenting symptoms, history of mechanical ventilation, foreign object aspiration, frequent infections, prematurity, vaccination history, number of household members, duration of breast feeding, parental consanguinity, family history of atopy or other chronic disorders, and exposure to tuberculosis) were recorded for each patient on forms. Physical examination findings and the results of routine and specific diagnostic tests (chest X-ray, purified protein derivatives (PPD) skin test, hemosiderin-laiden macrophages in broncho alveolar lavage , immunological evaluation, barium meal, gastroesophageal reflux scintigraphy, thoracic CT, and acid-fast staining of fasting gastric aspirate) were also recorded. Ethical clearance was taken from the Institutional Review Committee of Nepal Medical College Teaching Hospital. Data were taken from the patients' pro forma files. The data was analysed using SPSS version 16.

\section{RESULTS}

Among the 653 patients with a diagnosis of pneumonia during the study period, $74(11.3 \%)$ were diagnosed as RP. In this group, 47 (63.5\%) were male and 27 (36.4\%) were female (Fig. 1).

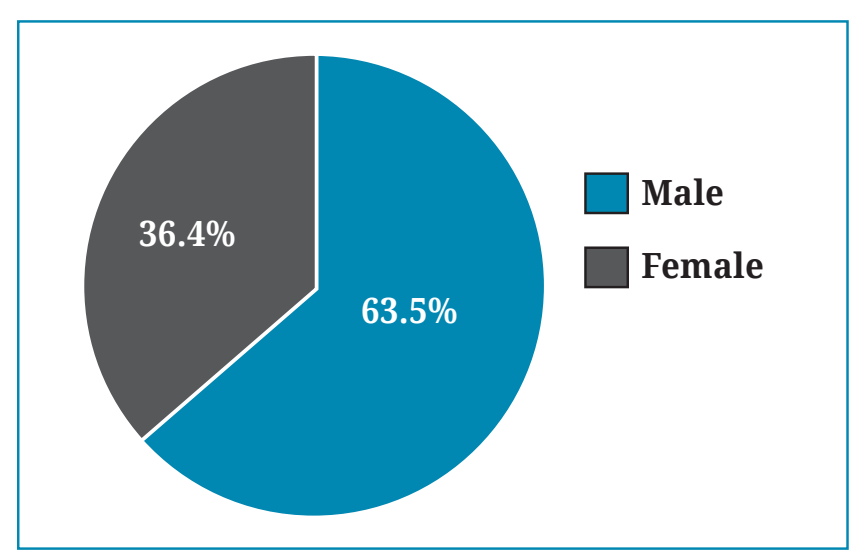

Fig. 1: Sex-wise distribution of recurrent pneumonia

The most common presenting symptom was cough in $98.3 \%$, followed by fever $63.5 \%$, fast breathing in $52.7 \%$ patients. On examination $52.7 \%$ had tachypnoea, $52.7 \%$ cases had crackles, $32.4 \%$ cases had wheezing, $51.3 \%$ cases had decreased breath sound, 10.8 cases had clubbing, and 1.3\% case had hepatosplenomegaly. Chest X-rays was done in all cases and in $49(66.2 \%)$ cases chest CT-scans was done. Chest X-ray showed consolidation in 43 (58.1\%), infiltration 17 (22.9\%) and lymphadenitis in $5.4 \%$ cases. CT scan chest revealed infiltration in $19(25.6 \%)$ cases, consolidation in $36(48.6 \%)$, bronchiectasis in $8(10.8 \%)$, air trapping in $7(9.4 \%)$, atelectasis in $5(6.7 \%)$, pleural effusion in $3(4.0 \%)$, interstitial lung disease in $1(1.3 \% \%)$ cases. Here, $16(21.6 \%)$ patients had aspiration syndrome out of which 6 (37.5\%) children had GERD and each 5 (31.2\%) children had cerebral palsy and foreign body aspiration.10 (13.5\%) patients had congenital heart disease, out of which $6(60.0 \%)$ children had VSD, 2 (20.0\%) patients had ASD and one of each had PDA 
and complex heart disease. Nine (12.1\%) patients had bronchial asthma. In each $8(10.8 \%)$ patients, they had immune deficiency disorders, bronchiectasis and congenital anomalies of respiratory system. In primary immune deficiency disorders $4(50.0 \%)$ children had common variable immunodeficiency, T-cell deficiency in $2(25.0 \%)$ patients and natural killer cell deficiency in 2 (25.0\%) patients. Congenital anomalies are an important underlying illness for recurrent pneumonia in children. Among congenital anomalies of respiratory system, 3 (37.5\%) patients had tracheoesophageal fistula, 1 (12.5\%) of each had tracheal stenosis, tracheal bronchus, agenesis of right pulmonary artery, hypoplasia of right lung, congenital cystoadenomatoid malformation. One $(1.3 \%)$ of each patient diagnosed as interstitial lung disease and pulmonary nocardiosis. No predisposing illness could be demonstrated in the remaining 9 (12.1\%) patients (Table-1).

\section{Table-1: Distribution of patients with RP according to underlying disorder/ risk factors.}

\section{Underlying Illness}

\section{Bronchial Asthma}

Bronchiectasis

Aspiration Syndrome

GERD

Cerebral Palsy

Foreign Body Aspiration

Total

Immunodeficiency

Primary

Common Variable

Immunodeficiency

$\mathrm{T}$ Cell deficiency

NK Cell deficiency

Secondary (HIV)

Total

\section{Congenital Heart Disease}

VSD

ASD

PDA

Complex Heart Disease

Total

Congenital Anomalies of Respiratory system

Tracheo-esophageal Fistula

Agenesis of right pulmonary artery

Hypoplasia of Right lung

Tracheal Bronchus

Tracheal Stenosis

Congenital cystoadenomatoid malformation

Total

Pulmonary Nocardiosis

Interstitial Lung Disease

Unidentified Cause

Total cases

\section{Recurrent Pneumonia}

$3(37.5 \%)$
No. of cases

9

8

$6(37.5 \%)$

$5(31.25 \%)$

$5(31.25 \%)$

16

\section{$\%$ of cases}

12.1

10.8

21.6

$8(66.66 \%)$

$4(33.33 \%)$

2 (16.66\%)

2 (16.66\%)

4 (33.33\%)

12

$6(60 \%)$

$2(20 \%)$

$1(10 \%)$

1 (10\%)

10

1 (12.5\%)

1 (12.5\%)

1 (12.5\%)

1 (12.5\%)

1 (12.5\%) 


\section{DISCUSSION}

The respiratory tract is protected by its local and general body defences. Nevertheless, respiratory tract infections constitute a major health problem all over the world. ${ }^{10}$ The etiology of RP varies depending upon the location of pneumonia, which can involve a single or various lobes or lung regions. Recurrent pneumonia involving a single lobe or segment, is caused by localized pathology, such as local compression, malformation or inflammation, whereas, pneumonias affecting more than one lobe, suggest a more generalized abnormality, such as mucociliary clearance dysfunction, aspiration, immunodeficiency or asthma. ${ }^{11}$ In our study, $11.3 \%$ of admission to the hospital with a diagnosis of pneumonia met the definition of recurrent pneumonia. It is slightly higher than other studies where $6.4 \%-9.0 \%$ of patients met the criteria for recurrent pneumonia. ${ }^{12,13}$

An underlying illness for recurrent pneumonia was found in $87.8 \%$ of our patients. Similar to Weigl et al data where cause for recurrent pneumonia was found in $83.0 \%$ of children. ${ }^{14}$ We did not identify the cause for RP in the remaining $12.1 \%$ of our patients. Comparatively, unknown aetiology of patients with recurrent pneumonia was found in $13.3 \%$ by Cabezuelo et al. ${ }^{15}$ The incidence and mortality rate of pneumonia in children are 10 times higher in developing countries than developed countries. Additionally, it is important to note that lack of epidemiological report from developing countries make it difficult to design global strategies for prevention, diagnosis, and treatment of RP. ${ }^{16}$

The most frequent underlying illness was aspiration syndrome found in $21.6 \%$ patients followed by congenital heart disease in $13.5 \%$ and bronchial asthma in $12.1 \%$ patients at our centre. Study done by Hovsing et al showed recurrent aspiration in $25.7 \%$ patients, lung disease (airway stenosis, bronchiectasis, middle lobe syndrome or tracheoesophageal fistula) in $16.1 \%$ patients and immune deficiency in $16.1 \%$ patients. ${ }^{17}$

Among the aspiration syndrome, gastroesophageal reflux was seen in $37.5 \%$ patients. Each $31.5 \%$ patients had oropharyngeal incoordination due to cerebral palsy and foreign body aspiration. These illnesses were confirmed by barium esophagography, milk scan and flexible bronchoscopy. Bolursaz et $a l^{18}$ showed aspiration syndrome in $51.7 \%$ patients among which GERD in $90.1 \%$ patients, cerebral palsy in $7.8 \%$ patients and foreign body aspiration in 3.9\% patients. Here, in $13.5 \%$ of patients with RP had congenital heart disease where Ventricular septal defect was most commonly diagnosed in $60.0 \%$ of children. Data analysed by Lodha et al ${ }^{19}$ revealed that $9.0 \%$ of children with RP had underlying congenital heart disease which is lower than our study. Bronchial asthma is an important underlying illness for recurrent pneumonia in children. In this study, $12.1 \%$ of children with bronchial asthma had RP. Despite recent advances in healthcare, many children referred with recurrent pneumonia are shown to have undiagnosed asthma. Asthma has been reported as an important underlying cause of recurrent pneumonia in children. ${ }^{20}$ In our study, $10.8 \%$ of the patients were diagnosed with primary immune deficiency disorders, including common variable immunodeficiency in $50.0 \%$ patients, T-cell deficiency in $25.0 \%$ patients and natural killer cell deficiency in $25.0 \%$ patients. Immune deficiency should be suspected in children with infections that are especially severe and recurrent, that are caused by unusual organisms, or that involve multiple sites in addition to the lungs. A study done by Çapanoglu et $a l^{21}$ showed that $4.0 \%$ children with $\mathrm{RP}$ had primary immunodeficiency which slightly lower than our data. This may be due to more number of referral cases in our centre.

Bilateral interstitial pneumonias on chest radiograph films and/or the presence of opportunistic infections were paramount in diagnosing HIV infection as an underlying factor in recurrent pneumonia. Similarly, HIV infection was found in $5.4 \%$ of children. ${ }^{22}$

Bronchiectasis was present in $10.8 \%$ cases. Past history of treatment for pulmonary tuberculosis was present in $62.5 \%$ cases while in $37.5 \%$. In cases predisposing factor could not be identified. A data of $60.7 \%$ cases of bronchiectasis were analysed by Pifferi et $a l^{23}$ which is very high as compared to our study. This may be due to less number of cases in our study.

Congenital anomalies are considered as an important underlying illness for recurrent pneumonia in children. We confirmed congenital anomaly of respiratory system in $10.8 \%$ of children. Three patients had tracheoesophageal fistula, one of each had tracheal stenosis, tracheal bronchus, agenesis of right pulmonary artery, hypoplasia of right lung, congenital cystoadenomatoid malformation. Congenital anomaly of respiratory system usually manifest during infancy as described by in this study. ${ }^{24}$ Patria et $a l^{25}$ analysed that the significant risk factors for recurrent pneumonia were GERD, a history of atopy and/or allergy, and asthma. In our study, aspiration syndrome and congenital heart disease were the important risk factors for recurrent pneumonia.

Our study had some limitations. As we included only hospitalized patients, the true number of patients with this condition may have been understimated.

In conclusion, recurrent pneumonia is still a diagnostic challenge in Pediatrics. Many children with RP need thorough investigations. In undiagnosed recurrent pneumonia, bronchial asthma, immune deficiency disorders, congenital heart disease, and gastroesophageal reflux should be considered in the differential diagnosis. Early treatment of the child's underlying condition is crucial in order to stabilize lung disease and thus prevent progressive pulmonary function deterioration. 


\section{REFERENCES}

1. World Health Organization. Global Tuberculosis Report 2016. Available from URL: http://www.who. int/tb/publications/global_report/en (Accessed: March 2017).

2. Wald E. Recurrent and non-resolving pneumonia in children. Semin Respir Infect 1993; 8: 46-58.

3. Lodha R, Puranik M, Natchu UC, Kabra SK. Recurrent pneumonia in children: Clinical profile and underlying causes. Acta Paediatr 2002; 91: 1170-3.

4. Owayed AF, Campbell DM, Wang EE. Underlying causes of recurrent pneumonia in children. Arch Pediatr Adolesc Med 2000; 154: 190-4.

5. Sheares BJ. Recurrent pneumonia in children. Pediatr Ann 2002; 31: 109-14.

6. Kumar M, Biswal N, Bhuvaneswari V, Srinivasan S. Persistent Pneumonia: Underlying Cause and Outcome. Indian J Pediatr 2009; 76: 1223-6.

7. Ozdemir O, Sari S, Bakirtas A, Zorlu P, Ertan U. Underlying diseases of recurrent pneumonia in Turkish children. Turk J Med Sci 2010; 40: 25-30.

8. Lodha R, Puranik M, Chandra U, Natchu M, Kabra SK. Persistent pneumonia in children. Indian Pediatr 2003; 40: 967-70.

9. McIntosh K. Community-acquired pneumonia in children. $N$ Engl J Med 2002; 346: 429-37.

10. Verhagen LM, de Groot R. Recurrent, protracted and persistent lower respiratory tract infection: A neglected clinical entity. J Infect 2015; 1: 106-11.

11. Mantzouranis E, Mathianaki K, Fitrolaki MD, Mihailidou E, Paspalaki P. A boy with recurrent pneumonia Breathe 2011; 7: 367-70.

12. Couriel J. Assessment of the child with recurrent chest infections. Br Med Bull 2002; 61: 115-32.

13. Çiftçi $E$, Güneş $M$, Köksal $Y$, İnce $E$, Doğru Ü. Underlying causes of recurrent pneumonia in Turkish children in a university hospital. $J$ Trop Pediatr 2003; 49: 212-5.

14. Weigl JA, Bader HM, Everding A, Schmitt HJ. Population-based burden of pneumonia before school entry in Schleswig-Holstein, Germany. Eur J Pediatr 2003; 162: 309-16.
15. Cabezuelo HG, Vidal MS, Abeledo GA, FronteraIzquierdo P. Underlying causes of recurrent pneumonia. Ann Pediatr 2005; 63: 40912.

16. Khaled S, Sherif AM, Kotb AM. Recurrent/Persistent pneumonia among Children in Upper Egypt. Mediterr J Hematol Infect Dis 2013; 5: 28.

17. Hoving MF, Brand PL. Causes of recurrent pneumonia in children in a general hospital. $J$ Paediatr Child Health 2013; 49: 208-12.

18. Bolursaz MR, Lotfian F, Ghaffaripour HF, Hassanzad M. Underlying causes of recurrent and persistent pneumonia in children at a pulmonary referral hospital in Tehran, Iran. Arch Iran Med 2017; 20: 266-9.

19. Lodha R, Kabra SK. Recurrent/persistent pneumonia. Indian Pediatrics 2000; 37: 1085-92.

20. Eigen H, Laughlin JJ, Homrighausen J. Recurrent pneumonia in children and its relationship to bronchial hypereactivity. Pediatri 1982; 70: 698704.

21. Çapanoglu M, Zorlu P, Sari E, Senel S. The etiology of recurrent pneumonia with onset during infancy, and the effect of risk factors on age at first episode and episode frequency. Turkish J Pediatr Dis 2017; 4: 243-7.

22. Beard LJ, Maxwell GM, Thong YH. Immunocompetence of children with frequent respiratory infections. Arch Dis Child 1981; 56: 101-5.

23. Pifferi M, Caramella D, Ragazzo V, Cangiotti AM, Macchia P, Boner AL. Bronchiectasis in children with recurrent pneumonia: an immunopathological damage associated with secondary ciliary dysmotility. Int J Immunopathol Pharmacol 2008; 21: 215-9.

24. Rubin BK. The evaluation of the child with recurrent chest infections. Pediatr infect Dis J 1985; 4: 88-98.

25. Patria F, Longhi B, Tagliabue C et al. Clinical profile of recurrent community- acquired pneumonia in children. BMC Pulm Med 2013; 10: 13-60. 